\title{
ERSCHEINEN UND LATENZ
}

\author{
ROBERTO WALTON
}

\begin{abstract}
Appearance and latency

The characteristics assigned by Heidegger to the coming-to-presence of what is present are examined in the light of Husserl's distinction between patency and latency. Subsequently, parallels are drawn regarding the notions of clearing and the unapparent. Finally, differences between Heidegger's openregion and Husserl's horizonality are shown.
\end{abstract}

In einem Manuskript von 1931, das den Titel Betrachtungen im Ausgang von der angeblichen Frage nach dem Sein des Seienden trägt, fragt sich Husserl, ob dieses Problem sich als berechtigt darstellen lässt: „Hat die heideggersche ,Ausarbeitung' der Frage nach dem, Sein des Seienden' einen guten Sinn, und welchen kann die von Aristoteles ausgehende Frage nach dem Seienden als Seienden überhaupt haben?"1. In diesem Aufsatz werde ich als Leifaden Themen von Heidegger nehmen, wie die Grundzüge des Anwesens des Anwesenden, die Welt als Lichtung, die Phänomenologie des Unscheinbaren und das Öffnende des Offenen, um zusammenlaufende Analysen von Husserl darzulegen. Trotz der Unterschiede lassen sich Parallelen ziehen in einem Versuch, einige Themen von Heidegger zu betrachten, ohne den Schritt zurück aus dem vorstellenden Denken in das andenkende Denken zu machen. Es ist zu beachten, dass Merleau-Ponty in einer Arbeitsnotiz von März 1959 auf das hinweist, was die Offenheit bei Husserl oder die Verborgenheit

1 Husserl Edmund, Zur phänomenologischen Reduktion. Texte aus dem Nachlass (1926-1935), hrsg. von Luft S., Dordrecht/Boston/London, Kluwer Academic Publishers, Husserliana XXXIV, 2002, S. $264 \mathrm{f}$. Creative Commons Attribution License (http://creativecommons.org/licenses/by/4.0). 
bei Heidegger besagen will. Er verbindet das Problem mit der „Struktur des Horizontes“ und dem „Überstieg zum Horizont“2.

\section{Das Anwesen des Anwesenden und der Kontrast Patenz/Latenz}

In der Vorlesung Was heisst Denken? hebt Heidegger als Grundzüge des Anwesens des Anwesenden die Unverborgenheit hervor, das Aufgehen aus ihr, das Eingehen zu ihr das Herbei- und das Hinweg ..., die Weile, die Versammlung, das Scheinen, die Ruhe und die verborgene Jähe des möglichen Abwesens. ${ }^{3}$ Die erste Gruppe von Grundzügen - die Unverborgenheit, das Aufgehen aus ihr, das Eingehen zu ihr, das Herbei- und das Hinweg ... - geht die Beweglichkeit der Anwesenheit an. Heidegger schreibt: „Aufgegangen aus der Unverborgenheit, ist das Anwesen auch schon eingegangen in das bereits Unverborgene [...]. Sein Anwesen ist aufgehendes Eingehen in das Unverborgene innerhalb der Unverborgenheit, $[\ldots]^{“ 4}$. Ein anwesendes Gebirge dient als Beispiel, wenn es auf sein Anwesen anstatt auf seine geologischen oder geographischen Merkmale hin betrachtet wird. Das Gebirge ist das Anwesende, d.h., das Seiende, das aus der Unverborgenheit aufgegangen und in die Landschaft eingegangen ist als das bereits Unverborgene. Nach Heidegger entfalten sich sowohl das Aufgehen aus der Unverborgenheit als auch das Eingehen in das Unverborgene innerhalb der Unverborgenheit.

2 Merleau-Ponty Maurice, Das Sichtbare und das Unsichtbare, übersetzt von Giuliani R. und Waldenfels B., München 1994, S. 239. K. Held macht uns in zahlreichen Aufsätzen auf „[d]ie verborgene Kontinuität des phänomenologischen Lebensweltdenkens im Übergang von Husserl zu Heidegger“ aufmerksam (Held Klaus, „Heidegger und das Prinzip der Phänomenologie“, in Gethmann-Siefert A. und Pöggeler O. (Hrsg.), Heidegger und die praktische Phänomenologie, Frankfurt a. M., Suhrkamp, 1988, S. 128). J. N. Mohanty weist auf einen strukturellen Isomorphismus hin: „By emphasizing the structural isomorphism between consciousness philosophy and existence philosophy, I have sought to draw attention to the often forgotten situation that 'the facts of the matter' in both are the same." (Mohanty J. N., The Possibility of Transcendental Phenomenology, Dordrecht/Boston/Lancaster 1985, S. 160). So G. Crowell spricht von einer inneren Kritik des transzendentalen Programms und betont die Möglichkeit einer Annäherung: „[...] 'parting of the ways' between the two phenomenologists makes better sense as an immanent criticism of Husserl's transcendental program rather than its wholesale rejection. It thus becomes possible to project a significant rapprochement between Husserl and Heidegger, one that leaves neither totally unrevised." (Crowell Steve Galt, Husserl, Heidegger, and the Space of Meaning. Paths Toward Transcendental Phenomenology, Evanston (Illinois), 2001, S. 181).

3 Vgl. Heidegger Martin, Was heisst Denken? (1951-1952), hrsg. von Coriando P.-L., Frankfurt am Main, Vittorio Klostermann, Gesamtausgabe 8, S. 239-242.

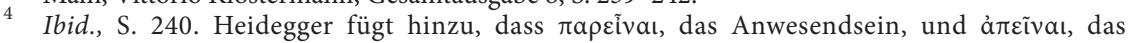
Abwesendsein, im griechischen عĩva auch mitgedacht sind. Deshalb sind das Herbei- und das Hinweg... Grundzüge des Anwesens. 
Hinsichtlich dieser ersten Gruppe der Grundzüge ergibt sich die Frage: Wie ist die Verflechtung vom Aufgehen aus der Unverborgenheit und Eingehen zu ihr zu verstehen? Die Frage richtet sich auch an das Herbei und das Hinweg, weil beide Grundzüge im Aufgehen und im Eingehen impliziert sind. Es fragt sich demnach, wie die Unverborgenheit beschaffen sein muss, um das Aufgehen und das Eingehen zu zulassen.

Man könnte von einem Paradox des Anwesens sprechen. Das Paradox besteht darin, dass das Anwesen des Anwesenden zugleich ein Aufgang aus der Unverborgenheit und ein Eingang zu der Unverborgenheit ist. Wenn Husserls Lehre des Horizontes, in welcher der Begriff von Patenz dem der Unverborgenheit entspricht, mit Heideggers Erörterung der Grundzüge verglichen wird, fallen uns sofort Parallelen und fundamentale Erklärungen auf. ${ }^{5}$ Husserl unterscheidet zwischen der patenten und der latenten Welt, und innerhalb der patenten Welt hält er die unthematische Welt und die thematische Welt als Welt der aktuellen Intentionen auseinander. M.a.W.: eine unthematische Patenz kennzeichnet den nicht im Zentrum der Aufmerksamkeit stehenden Außenhorizont. Thematische Patenz hat das Gebirge, auf das wir achten, wie das Luberon-Gebirge, das im Seminar in Le Thor 1969 als Beispiel eines sich von sich selbst her zu sehenden Phänomens erwähnt wird. ${ }^{6}$ Unthematische Patenz hat die übrige Gebirgskette im Hintergrund des Wahrnehmungsfeldes. In der Latenz bleibt das, was nicht zu diesem Feld gehört. Andererseits unterteilt sich die latente Welt in den Horizont der Bekanntheit oder typischer Vertrautheit, der durch die Sedimentierung früherer Erfahrungen gebildet wird, und in den Horizont der Unbekanntheit. Der Horizont der Bekanntheit ist ein Horizont von leeren Intentionen. Diese können durch Vergegenwärtigungen, die einen sekundären Sinn der Patenz mitbringen, erfüllt werden. Husserl fasst diese Unterscheidungen im folgenden Text zusammen:

Danach ist die Rede von Horizont doppelsinnig: Fürs Erste der Horizont, der zum jeweilig Patenten gehört, total genommen, die entsprechende Latenzsphäre; fürs Zweite das in den aktiven Intentionen Bewusste, der jeweilige Bereich des Thematischen, hat seinen unthematischen Horizont. Wir haben also darin Patentes aber Unthematisches und Latentes Unthematisches. ${ }^{7}$

5 „This theme of absence was, I believe, a stimulus for Heidegger's notion of unconcealedness as involved in truth." (Sokolowski Robert, Introduction to Phenomenology, Cambridge, Cambridge University Press, 2008, S. 217).

6 Vgl. Heidegger M., Seminare (1951-1973), hrsg. von Ochwadt C., Frankfurt am Main, Vittorio Klostermann, Gesamtausgabe 15, 1986, S. 327 f.

7 Husserl E., Manuskript D 14, 36 b. Dem Husserl-Archiv zu Leuven gilt mein Dank für die freundliche Erlaubnis, aus unveröffentlichen Manuskripten Husserls zu zitieren. 
Die Doppelsinnigkeit des Horizontes bringt die vielfältige Unterscheidung zwischen der thematischen Patenz, der unthematischen Patenz, der bekannten Latenz und der unbekannten Latenz mit sich. Der Begriff der Unverborgenheit dreht sich nach Husserl um die thematische Patenz und kann enger oder weiter gefasst werden, je nachdem, ob sich die Unverborgenheit auf den Bereich der unthematischen Patenz begrenzt oder auf denjenigen der bekannten Latenz ausbreitet. Mit dieser Unterscheidung sind wir fähig zu verstehen, was Heidegger das Aufgehen aus der Unverborgenheit, das Eingehen zur Unverborgenheit und das Unverborgene innerhalb der Unverborgenheit nennt. Zur Aufklärung dieser Schwierigkeit ist das Anwesen des Anwesenden auf zweierleie Weise zu betrachten.

Einerseits handelt es sich um das Aufgehen aus der unthematischen Patenz und das Eingehen zur thematischen Patenz. In diesem Fall entspricht das Unverborgene innerhalb der Unverborgenheit der thematischen Patenz innerhalb der unthematischen Patenz. Andererseits geht es um das Aufgehen aus der bekannten Latenz und das Eingehen zur Patenz überhaupt, so dass das Unverborgene innerhalb der Unverborgenheit der Patenz innerhalb der bekannten Latenz entspricht.

Demzufolge kann das Aufgehen aus der Unverborgenheit als ein Erscheinen aus der unthematischen Patenz oder aus der bekannten Latenz verstanden werden. Korrelativ ist das Eingehen zur Unverborgenheit als ein Ankommen in der thematischen Patenz oder in der Patenz als solcher zu erfassen. Dies bedeutet, dass das Unverborgene innerhalb der Unverborgenheit als die thematische Patenz, die gegenüber der Patenz im großen und ganzen deutlich hervortritt, oder als die Patenz überhaupt, die sich von der Latenz im Großen und Ganzen abhebt, gesehen werden kann.

\section{Zwei Sinne der Unverborgenheit}

Das erwähnte Paradox des Unverborgenen, das aus der Verflechtung zwischen Aufgehen und Eingehen erwächst, lässt sich auflösen, weil die vermeintliche Widersinnigkeit darin besteht, dass Heidegger zwei Sinne der Unverborgenheit zusammengefasst hat.

i)

Eingehen zur Unverborgenheit = zur thematischen Patenz (das Herbei)

$=$ das Unverborgene innerhalb 
Aufgehen aus der Unverborgenheit

$=$ aus der unthematishen Patenz

(das Hinweg)

$=$ der Unverborgenheit

ii)

Eingehen zur Unvervorgenheit
= zur Patenz überhaupt
(das Herbei)

Aufgehen aus der Unverborgenheit

$=$ aus der bekannten Latenz

(das Hinweg)
$=$ das Unverborgene

innerhalb

$=$ der Unverborgenheit

Heideggers Beschreibung des Anwesens des Anwesenden durch die Erscheinung des Gebirges innerhalb der Landschaft ermöglicht nicht nur diesen Hinweis auf Husserls erklärende Analyse, sondern zeigt die Rolle eines Subjekts, dem das Gebirge und die Landschaft erscheinen. Die Unterscheidung zwischen thematischer Patenz und unthematischer Patenz setzt ein Subjekt voraus, das seine Aufmerksamkeit auf ein Thema richtet. Und die Unterscheidung zwischen bekannter und unbekannter Latenz hat ein mit einer typischen Welthabe ausgestattetes Subjekt als Voraussetzung.

Die zweite Gruppe der Grundzüge - die Weile, die Versammlung, das Scheinen, die Ruhe - betrifft die Struktur der Anwesenheit. In dieser Hinsicht ist es möglich, eine gewisse Ähnlichkeit zu zeigen. Aber diese Züge können hier übergangen werden, weil sie nicht so wichtig für unser Thema sind. ${ }^{8}$

Der letzte von Heidegger hervorgehobene Grundzug - die verborgene Jähe des stets möglichen Abwesens in die Verborgenheit - bezieht sich auf die Kehrseite der Anwesenheit. Das Aufgehen entfaltet sich in einer ursprünglichen Einheit zugleich

8 Zum Beispiel zeigt sich eine Ähnlichkeit im Folgenden. Das Anwesen besagt nicht nur das Hervorkommen, sondern auch das Währen in die Unverborgenheit. Deshalb ist das Anwesende das Währende, d.h. was in die Unverborgenheit hineinwährt. Auf Grund dieses Verweilens ist die Weile als ein Grundzug zu betrachten: „[...] Anwesen <ist> je das Eingehen in eine Weile von Unverborgenheit. [...] Weilen ist das ruhende, zum Stehen gekommene Vorkommen, nämlich vor in die Unverborgenheit des Vorliegenden." (Heidegger M., Was heisst Denken?, op. cit., S. 241). Der Weile entspricht bei Husserl die aktuelle Fortdauer, die sich im aktuellen Jetzt konstituiert und eine abgelaufene Strecke vom Anfangspunkt bis zum Jetztpunkt sowie eine übrige Strecke von Jetztpunkten hat. Im Gegensatz zur erledigten Dauer, die ihr Ende hat und so nicht mehr aktuelle Wirklichkeit ist, hat diese nicht abgeschlossene Dauer vor sich die noch ungewordene Zukunft, die sich der Gegenwart nähert. Vgl. Husserl E., Analysen zur passiven Synthesis. Aus Vorlesungsund Forschungsmanuskripten (1918-1926), hrsg. von Fleischer M., Den Haag, Martinus Nijhoff, Husserliana XI, 1966, S. 416 f. 
als das Insichzurückgehen im Sinne der Verbergung: „Weil Sein heißt: aufgehendes Erscheinen, aus der Verborgenheit heraustreten, deshalb gehört zu ihm wesenhaft die Verborgenheit, die Herkunft aus ihr" ${ }^{\text {"9 }}$. Heidegger spricht von der nächsten Nachbarschaft von Aufgehen und Verbergen, weil Entbergung und Verbergung "nicht als zwei verschiedene, nur aneinander geschobene Geschehnisse, sondern als Eines und das Selbe " 10 zu verstehen sind. Umgekehrt bedürfte die Verborgenheit der Unverborgenheit. Dies bedeutet, dass das Geheimnis offenbar sein muss: „Wenn das Wesen der Wahrheit in der Offenbarkeit des Seienden zu suchen ist, dann erweist sich die Verborgenheit und Verhüllung als eine eigene Weise der Offenbarkeit"11.

Husserls Analysen stimmen mit denen Heideggers darin überein, dass jedes Erscheinen aus einer Unverborgenheit erfahren werden muss, weil das Unverborgene sich aus ihr ernährt. So fassen Husserl und Heidegger beide eine unvermeidliche Voraussetzung für jede Enthüllung auf. In ähnlicher Weise bemerkt Husserl auch, dass jede Auslegung der dunklen Dimension der Intentionalität von der gegenwärtigen Erfahrung ausgeht und sich in der Entfaltung der Horizonte und in der Rückkehr zum Vor-Ich und seiner Vor-welt erweitert.

Die Verborgenheit ist gerade der Grundzug, den Merleau-Ponty mit Husserls Offenheit verbindet. Sie wird von Husserl als reiner Horizont der Latenz dargestellt, innerhalb dessen weitere Scheidungen möglich sind. Der Horizont der Bekanntheit mit seinen Leerintentionen lässt gerade Klarheit und Deutlichkeit zu. Während eine zunehmende Klarheit von der Erfüllung der Verweisungen abhängt, erreicht eine abnehmende Deutlichkeit einen Punkt, wo alle Verweisungen verschwinden und sich damit aller Inhalt auflöst. Deshalb unterscheidet Husserl zwischen dem Horizont der leeren Vorstellungen und dem leeren, unterschiedlosen Horizont. Der letzte hat „den Charakter der Potentialität für eine Mannigfaltigkeit von zugehörigem Sonderbewusstsein, von abgegrenzten intentionalen Erlebnissen, $[\ldots]^{\text {"12 }}$. Husserl versucht, ein Miteinander und Ineinander von Horizonten und eine Verweisung von an sich ersten auf an sich spätere Horizonte auszulegen. Demzufolge ist die Welt als universaler Horizont von der Horizonthaftigkeit jedes

9 Heidegger M., Einführung in die Metaphysik, hrsg. von Jaeger P., Frankfurt am Main, Vittorio Klostermann, Gesamtausgabe 40, 1983, S. 122.

10 Heidegger M., Vorträge und Aufsätze (1936-1953), hrsg. von Herrmann F.-W. von, Frankfurt am Main, Vittorio Klostermann, Gesamtausgabe 7, 2000, S. 277.

11 Heidegger M., Hölderlins Hymnen „Germanien“ und „Der Rhein“, hrsg. von Ziegler S., Frankfurt am Main, Vittorio Klostermann, Gesamtausgabe 39, 1980, S. 119. „Ein Geheimnis, das in seiner verhüllenden Macht nicht gewusst wird, ist keines. Je höher das Wissen um die Verhüllung und je echter das Sagen von ihr als solcher, um so unberührter bleibt seine verbergende Macht." (Idem).

12 Husserl E., Zur Phänomenologie der Intersubjektivität. Texte aus dem Nachlass. Zweiter Teil: 1921 - 1928, hrsg. von Kern I., Husserliana XIV, Den Haag, Martinus Nijhoff, Husserliana XIV, 1973, S. 150. 
erfahrenen Objekts zu entüllen: „Diese unter dem Titel Welterfahrung - transzendentale Welterfahrung - vollzogene Leistung impliziert in Verborgenheit eine Stufenfolge von Leistungen, in denen die Totalleistung (diejenige, wodurch Welt ,für mich ist') fundiert ist, $[\ldots]^{\text {"13 }}$.

Heidegger verbindet die Verborgenheit, aus der das Aufgehen entspringt, mit einem Übermaß. Hinsichtlich dieser Überfülle schreibt er: „Daher ist immer die Dimension des gänzlichen Übermäßigen hervorzuheben, der die Philosophie entspringt" 14 . Seinerseits spricht Husserl von einem den Horizonten innewohnenden Überschuss, aber der Akzent liegt bei ihm auf einer ins Unendliche fortschreitenden Erfahrung, die sich ,auf einen idealen Pol als Korrelat einer in der Form , in infinitum 'inszenierte Vorstellung der frei möglichen Fortführung einstimmiger Erfahrung, [...]"15 richtet. Hier liegt ein wesentlicher Unterschied, weil Husserl auf eine verborgene Idee hinweist, die durch Reflexion entwickelt werden kann. Husserl geht einen Schritt weiter, indem er nicht nur die Verborgenheit als eine Rücklage für die Unverborgenheit und diese als die Bekundung einer verhüllenden Macht versteht, sondern die Verborgenheit selbst als die Potentialität begreift, die den ersten Anstoß zur Idee gibt. ${ }^{16}$ Auf Grund der offenen Unendlichkeit der Erfahrung wird die Idee der Allheit der Erfahrung aufgebaut. Wie die Verminderungsreihe in einem Nullpunkt mit dem Verschwinden des Erscheinens endet, so zielt die Steigerungsreihe von der Fülle des Gegebenen auf ein Maximum des Erscheinens. Husserl spricht von einem Steigerungsbewusstsein, „in dem die betreffende Idee als eine evident motivierte Antizipation [...] selbstgegeben ist" 17 . Trotz ihrer Geschlossenheit ist die endliche Erfahrung grenzenlos beweglich. Deshalb weist Husserl auf „eine unendliche Welt als Idee, die wir im Gang der ständig sich wandelnden Endlichkeiten als unendlich fernen Pol ins Auge bekommen"18. Eine Genesis der Idee findet auf Grund des Horizontbewusstseins in einer Reihe von Schritten statt. So stufen sich die Auslegung

Husserl E., Späte Texte über die Zeitkonstitution (1929-1934). Die C-Manuskripte, hrsg. von Lohmar D., Dordrecht, Springer, Husserliana-Materialien VIII, 2006, S. 4.

14

15

16 K. Held hebt hervor, dass die Möglichkeit einer theoretischen Neugier auf die Unendlichkeit der Welt im Modus der Eigentlichkeit durch Heidegger nicht ernstlich berücksichtigt worden ist. Vgl. Held K., „Die Endlichkeit der Welt. Phänomenologie im Übergang von Husserl zu Heidegger“, in Niemeyer B. und Schütze D. (Hrsg.), Philosophie der Endlichkeit, Würzburg, Königshausen \& Neumann, 1992, S. 143 f.
17 Husserl E., Analysen zur passiven Synthesis, op. cit., S. 202 f.
18 Husserl E., Die Krisis der europäischen Wissenschaften und die transzendentale Phänomenologie, hrsg. von Biemel W., Den Haag, Martinus Nijhoff, Husserliana VI, 1962, S. 500 f. 
der Horizonte, die Wiederholung dieser Enthüllung von Latentem, das durch Reflexion erreichbare Bewusstsein von einer Richtung und die folgende Ideation des Pols dieses Gerichtetseins ab. Die mögliche Unendlichkeit des Erscheinens ist die Bedingung der Idee und diese tritt aus einer Extrapolation heraus, die sich auf die fortschreitende Auslegung von Horizonten gründet. Diese ideal ausgestaltete Konzeption ist im Bewusstsein des Fortschreitenkönnens fundiert und bedeutet das Ineinsgreifen oder Übergreifen der Allheit des prozesshaft sich Kettenden. ${ }^{19}$ So bringt Husserl das Erscheinen in Verbindung mit einer Verbergung der ihm innewohnenden Idee.

\section{Die Lichtung und die Weltvorstellung}

Im Zusammenhang mit der Unverborgenheit steht nach Heidegger die Lichtung, d.h. das Offene, das dem Anwesenden ein mögliches Scheinenlassen erlaubt. Wie bekannt ist, verweist Heidegger bei dem Wort „Lichtung“ auf das französische Wort „clarière“ und verbindet es mit der Waldlichtung, d.h. einem von Bäumen freien Platz, wo das Anwesende zum Vorschein kommen kann: „Die Lichtung ist das Offene für alles An- und Abwesende " 20 . Heidegger spricht von Welt in diesem Zusammenhang: „,Welt ' bedeutet [...] die Offenheit des Seins. [...] ,Welt' ist die Lichtung des Seins, in die der Mensch aus seinem geworfenen Wesen her heraussteht" ${ }^{\text {"21. }}$ Nach Heidegger gibt es eine gelichtete Dimension, aus der das ekstatische Innestehen des Menschen in der Wahrheit des Seins und das geworfene Herausstehen des Menschen aus dem Wurf des Seins sich entfalten. Da die Lichtung das Innestehen und das Herausstehen umfasst, ist sie eine vermittelnde Dimension zwischen Mensch und Sein. Sie ist die Dimension, die „das ,Zwischen ' lichtet“"22, so dass die Verborgenheit ins Offene kommt, ohne zu verschwinden. ${ }^{23}$

Heideggers Lichtung des Seins kann mit Husserls Begriff von Weltvorstellung in Verbindung gebracht werden. Sowie die Auslegung eines Einzeldinges sein

19 Vgl. Husserl E., Manuskript A VII 21, 3b-6a.

20 Heidegger M., Zur Sache des Denkens (1962-1964), hrsg. von Herrmann F.-W. von, Frankfurt am Main, Vittorio Klostermann, Gesamtausgabe 14, 2007, S. 81.

21 Heidegger M., Wegmarken (1919-1961), hrsg. von Herrmann F.-W. von, Frankfurt am Main, Vittorio Klostermann, Gesamtausgabe 9, 1976, S. 350.

22 Idem.

23 „Die Lichtung der Verbergung meint nicht die Aufhebung des Verborgenen und seine Freistellung und Umwandlung ins Unverborgene, sondern gerade die Gründung des abgründigen Grundes für die Verbergung [...]." (Heidegger M., Beiträge zur Philosophie (Vom Ereignis) (1936-1938), hrsg. von Herrmann F.-W. von, Frankfurt am Main, Vittorio Klostermann, Gesamtausgabe 65, S. 352). 
Sosein hervorbringt, macht die Weltvorstellung deutlich, was in der strömenden, verschwimmenden Welterfahrung impliziert ist: „Hätte ich sie nicht gebildet, so wüsste ich nichts von der Welt. [...] Das Wort ,Welt ' hat seine Wortbedeutung nur dadurch, dass zum Ausdruck gebracht wird, was diese Vorstellung beibringt " ${ }^{24}$. In dieser Hinsicht ist hervorzuheben, dass die Weltvorstellung die Gegebenheitsweise der Welt selbst ist, weil die Spannung zwischen Weltvorstellung und wirklicher Welt vom transzendentalen Standpunkt verschwindet. ${ }^{25}$ Die Weltvorstellung ist das Korrelat eines thematischen Weltbewusstseins im Kontrast zu der unthematischen Welt als universalem Horizont. Was wir ständig haben, ist ein unthematisches Bewusstsein von der Welt, das mit jedem Thema einhergeht. Die schlichte Welterfahrung ist noch nicht eine Weltvorstellung, weil diese einen Aufbau fordert, der sich auf „eine universale Bewegung und Synthese in der Bewegung aller meinen Vorstellungen ${ }^{\text {"26 }}$ beläuft. Es handelt sich um eine Synthese, die alle meine Vorstellungen zur Einheit einer Welt zusammenfasst.

Mit diesem Aufbau der Vorstellungen gliedert sich das Weltbewusstsein in zwei verschiedene Richtungen. Husserl schreibt: „Dadurch bedeuten Weltvorstellung und Welterfahrung für das Ich Bewusstsein der für es seienden Welt, [...]“27. Im Gegensatz zur Welterfahrung, die gleichbedeutend mit der Verbergung ist, hat die Weltvorstellung einen gelichteten Bereich zur Folge.

Weltvorstellungen werden als einheitliche Spielräume von Möglichkeiten derselben Welt auf dem Grund der Bedingungen unserer Umgebung aufgebaut und wiederaufgebaut. Als vorgestellte wird die Welt als ein „Entwurf“ 28 konstruiert, der sich nicht auf die unmittelbaren Möglichkeiten der Umgebung beschränkt. Die Möglichkeiten können auf verschiedene Weise wiederaufgebaut werden, weil die Umwelt, die der Weltvorstellung zugrundeliegt, sich nach den jeweiligen praktischen Interessen verwandelt. Einzelmenschliche und gemeinschaftliche Weltvorstellungen werden als die geltenden Umwelten entfaltet. Sie können deutlicher durch Aufwicklungen, konkreter durch einen Zuwachs an Sinnschichten und klarer durch Anschauungen gestaltet werden. Durch Erfüllung lernen wir das kennen, was in den Horizonten liegt. Die Klärung versucht deshalb eine Erläuterung durch exemplarische Anschauungen. Husserl betont, dass eine aktuelle und

\footnotetext{
Husserl E., Die Lebenswelt, op. cit., S. 76.

Vgl. Husserl E., Erste Philosophie (1923/24). Zweiter Teil: Theorie der phänomenologischen Reduktion, hrsg. von Boehm R., Haag, Martinus Nijhoff, Husserliana VIII, 1959, p. 480.

26 Husserl E., Die Krisis der europäischen Wissenschaften und die transzendentale Phänomenologie. Ergänzungsband aus den Nachlass 1934-1937, hrsg. von Smid R. N., Dordrecht/Boston/London, Kluwer Academic Publishers, Husserliana XXIX, 1993, S. 268.

27 Ibid., S. 253.

28 Husserl E., Die Lebenswelt, op. cit., S. 76.
} 
anschaulich durchgeführte Weltvorstellung die Grundlage unseres Wissens von der Welt ist: „[...] wenn wir über Welt nachdenken, so leitet uns die ,Vorstellung «Welt»" nicht als Einheit bloßer Erfahrung, sondern der ,altbekannte,, in Vagheit auftauchende“ Begriff, Welt “'29. Demnach ist die Weltvorstellung eine vermittelnde Dimension zwischen der dunklen Welterfahrung und dem erfahrenden Ich, das sich in der Welt zurechtzufinden sucht, so wie die Lichtung eine vermittelnde Dimension zwischen Sein und Dasein ist.

Nach Heidegger „zieht durch die Lichtung ein ständiges Verbergen in der Doppelgestalt des Versagens und des Verstellens " ${ }^{30}$. Das Verbergen ist ein Versagen, wenn das Seiende außerhalb des Gelichteten bleibt, und es ist ein Verstellen, wenn das Seiende durch ein anderes Seiendes innerhalb des Gelichteten verschleiert wird. In Bezug auf die letzte Art des Verbergens schreibt Heidegger: „Ein be-

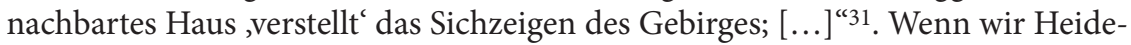
ggers Unterscheidung von zwei Arten des Verbergens mit der oben dargestellten Gegenüberstellung von Patenz und Latenz vergleichen, dann fallen uns einige Parallelen auf. Husserl entwickelt etwas Ähnliches, indem er das Versagen als den Ausschluss aus der Weltvorstellung und das Verstellen als die Verleihung einer untergeordneten Stellung in der Weltvorstellung bezeichnet. So wie die Gestalt des Versagens zur Lichtung gehört, ist die Weltvorstellung durch das Herausfallen in die schlichte Welterfahrung charakterisiert. Und so wie die Gestalt des Verstellens zur Lichtung gehört, ist die Weltvorstellung durch Überordnung und Unterordnung der eingeschlossenen Möglichkeiten gekennzeichnet.

\section{Das Unscheinbare und das Unerfahrbare}

Im Protokoll seines letzten Seminars in Zähringen wird berichtet, dass Heidegger das Thema des ursprünglichen Sinnes der Phänomenologie behandelt. Er bemerkt dazu, dass „die Phänomenologie ein Weg <ist>, der hinführt vor ... und sich das zeigen lässt, wovor er geführt wird. Diese Phänomenologie ist eine Phänomenologie des Unscheinbaren“32. Das Thema tritt auf im Insichzurückgehen, im Zu-

\footnotetext{
Ibid., S. 70.

Heidegger M., Holzwege (1935-1946), hrsg. von Herrmann F.-W. von, Frankfurt am Main, Vittorio Klostermann, Gesamtausgabe 5, 1977, S. 41.

31 Heidegger M., Parmenides, hrsg. von Frings M. S., Frankfurt am Main, Vittorio Klostermann, Gesamtausgabe 54, 1982, S. 54.

32 Heidegger M., Seminare, op. cit., S. 399.
} 
rücknehmen und Zurückhalten, welches das Aufgehen kennzeichnet. Heidegger macht klar, dass das Aufgehen nicht innerhalb des Aufgehenden vorkomme und „das anfänglich Gesichtete, das, obzwar zunächst und zumeist, ja oft überhaupt nie eigens Erblickte" ${ }^{\text {"33 }}$ sei. Das Aufgehen erweist sich als das Gesichtete im Sinne des in gewisser Weise mit dem Blick Verfolgten, aber nicht als das Erblickte im Sinne des Erreichten in seinem Insichzurückgehen. Die Situation ist ähnlich derjenigen, in welcher der Raum in der Einrichtung eines Zimmers und die Zeit in den Ziffern und der Zeigerstellung der Uhr gesichtet, aber nicht erblickt werden. ${ }^{34}$ Wir haben gesehen, dass das Anwesen und die Lichtung keine Aufhebung des Verborgenen durch eine Umwandlung ins Unverborgene einschliessen.

Bei Husserl treffen wir in allen Bereichen der Erfahrung auf einen Überschuss, der sich in das Erfahrenen eingräbt. Etwas ist gesichtet aber nicht erblickt, wie Heidegger sagt. Was die Welt angeht, zeigt sich der leere Horizont als „das Fernfeld der offenen Unbekanntheiten“35 oder „der unbestimmt offene Randhorizont“36 und „umspannt eigentlich die ganze Welt und auch sie als einen Horizont, einen unendlichen Horizont möglicher Erfahrung “37. Hinsichtlich des Objektes spricht Husserl „von einem intentionalen Leerhorizont“38 und einem „Spiel von Gewinn und Verlust“"39, weil die fortschreitende Erfüllung der Horizonte zugleich eine fortschreitende Entleerung ist. Entscheidend für Husserl ist, dass dieses Spiel sich nicht dem Fortschritt in der Erkenntnis widersetzt. Da die entgehenden Dingerscheinungen andere Erscheinungen als die aufgehenden sind, bedeutet das Spiel ein Gewinn an dauernder Kenntnis, die „wie aus einer Vorratskammer wieder herausgeholt werden und zur Verknüpfung mit anderen solchen Erwerben verar-

33 Heidegger M., Heraklit, hrsg. von Frings M. S., Frankfurt am Main, Vittorio Klostermann, Gesamtausgabe 55, 1979, S. 143.

34 ",Raum ${ }^{\prime}$ und ,Zeit` sind jedesmal das Unscheinbare, aber gleichwohl Gesichtete, nur nicht das Erblickte und vordergründig Gegenständliche." (Idem.).

35 Husserl E., Phänomenologische Psychologie. Vorlesungen Sommersemester 1925, hrsg. von Biemel W., Den Haag, Martinus Nijhoff, Husserliana IX, 1962, S. 94. Husserl E., Zur Phänomenologie der Intersubjektivität. Texte aus dem Nachlass. Dritter Teil: 19291935, hrsg. von Kern I., Den Haag, Martinus Nijhoff, Husserliana XV, 1973, S. 209. Husserl E., Erste Philosophie. Zweiter Band, op. cit., S. 148. Das athematische Welbewusstsein ist nicht nur ein Randhorizont, in dem es keine zu Gegenständen orientierte Intentionen gibt, sondern auch ein Boden für die thematische Gegenstandsrichtung, d.h., für den „Wechsel und Umschlag von Sein und Schein" in den Korrekturen der Erfahrung. Vgl. Fink Eugen, VI. Cartesianische Meditation. Teil 2: Ergänzungsband, hrsg. von Kerckhoven G. van, Dordrecht/Boston/London, Kluwer Academic Publishers, Husserliana-Dokumente II/2, 1988, S. 90 f. Husserl E., Analysen zur passiven Synthesis, op. cit., S. 6.

Husserl E., Phänomenologische Psychologie, op. cit., S. 434. 
beitet werden kann “40. In Husserls Horizont der Latenz bildet diese Kammer den erwähnten Bereich der Bekanntheit. ${ }^{41}$

Objekte werden aus einem Hintergrund, der unerfahren bleibt, herausgehoben. Im Gesamtbereich der Affektion gibt es eine Gradualität der Lebendigkeit, auf Grund deren Husserl auf „ein gewisses Merklichkeitsrelief“ hinweist. Zu diesem Relief gehört „ein Hintergrund von Unlebendigkeit, von affektiver Wirkungslosigkeit“, der endlich in das Unbewusste als der Nullpunkt der Merklichkeit gerät. Dieser Nullpunkt, so erklärt Husserl, ist „keineswegs ein Nichts“ ${ }^{42}$. Es handelt sich nur um ein Nichts an affektiver Kraft, aber nicht um eine Null an der Intensität qualitativer Momente. Was im Hintergrund liegt, kann unter günstigen Umständen zur Abhebung kommen, weil es eine Tendenz zur Affektion als Potentialität der Affektion enthält. ${ }^{43}$

Der wichtigste Fall des Unscheinbaren ist bei Heidegger das Ereignis. Hier ist zu zeigen, dass die Strukturparallelität sich nicht auf die Weltvorstellung und die Lichtung als vermittelnde Dimension begrenzt. Der Isomorphismus beginnt mit den vermittelten Momenten. Ebenso es eine Korrelation zwischen der Welt und der Subjektivität bei Husserl gibt, schildert Heidegger eine Art Korrelation in der wechselseitigen Beziehung von Sein und Dasein. ${ }^{44}$ Heidegger spricht über das Ereignis als die Mitte zwischen dem Zuruf oder Zuspruch des Seins und der Entsprechung oder Zugehörigkeit des Daseins. Sein und Dasein sind einander in einem Zusammengehören übereignet. Das Sein braucht das Offene einer Lichtung

40

Vor der Entfaltung der Theorie der Horizontintentionalität wird die Gegebenheitsweise des leeren Raumes behandelt. Husserl schreibt: „Eine Körperlichkeit ist gesehen, aber unendlich viele Möglichkeiten für weitere Körperlichkeiten lässt sie offen, nämlich in dem ,Zwischen', [...]. Gesehen sind die Körper, und mit dem Gesehenen erfasst ist das Zwischen, [...] Der Raum ist also eher mitgesehen." (Husserl E., Ding und Raum. Vorlesungen 1907, hrsg. von Claesges U., Den Haag, Martinus Nijhoff, Husserliana XVI, 1973, S. 261 f.). Über Husserls Überlegungen zum leeren Raum vgl. Figal Günther, Unscheinbarkeit. Der Raum der Phänomenologie, Tübingen, Mohr Siebeck, 2015, S. $48-52$.

Husserl E., Analysen zur passiven Synthesis, op. cit., S. 167.

Hinsichtlich dieser Vor-Objekte spricht Husserl „von einem Null-Grad der Affektion [...] <einem> Limesfall, der der Reizkraft Null, [...]“ und er erklärt: „Aber aus dieser ,Nacht', dieser leeren Stelle können jeweils Stimmen vorbrechen dadurch, dass die Null-Kraft einer Affektionskomponente Kraftzuwachs erfährt.“ (Husserl E., Späte Texte über die Zeitkonstitution, op. cit., S. 191 f.).

"The relations between Heidegger's and Husserl's conceptions of worldhood remain to be explored, and this would be an important project for twenty-first century Heidegger studies. Finally, it is important to recognise that Heidegger, as much as Husserl, sought to think through the relation that Husserl calls the ,noetic-noematic correlation. Heidegger as a phenomenologist and as a transcendental philosopher (although he eventually abandons the language of transcendental philosophy) remains committed to the essential a priori correlation between Dasein and Sein". (Moran Dermot, „Dasein as Transcendence in Heidegger and the Critique of Husserl“, in Georgakis T. und Ennis P. J. (Hrsg.), Heidegger in the Twenty-First Century, Dordrecht, Springer, 2015, S. 44). 
und das Dasein ist offen für das Sein..$^{45}$ Da das Ereignis als Unzeigbares im Verborgenen bleibt, können wir es nicht als ein Gegenüber vor uns stellen. Es ist nur „erblickbar" 46 durch einen Einblick in die Gaben seines Ereignens. Da das Ereignis das Anwesen in sein Eigenes als Gabe seines Ereignens bestimmt, das An- und Abwesende in sein jeweiliges Eigenes erbringt und Menschen und Sein einander übereignet, kann Heidegger sagen: „Es gibt nicht anderes, worauf das Ereignis noch zurückführt, woraus es gar erklärt werden könnte. [...] Das Ereignis ist das Unscheinbarste des Unscheinbaren, [...]“47.

Bei Husserl begegnen uns ebenfalls derartige Gedanken. Als „Ursein oder Urgeschehen“ ist die lebendige Gegenwart „das ,Urphänomen', auf das alle transzendentale Rückfrage in der Methodik der phänomenologischen Reduktion“ 48 zurückgeleitet wird. Während das Ereignis sich entbirgt oder entzieht, bleibt das urtümliche Strömen der lebendigen Gegenwart bei Husserl immer außerthematisch: „Es ist als Vor-Sein unerfahrbar, unsagbar; [... $]^{“ 49}$. Hier kann eine methodische Übereinstimmung, die in zwei Schritten zu etwas Letztem zurückführt, unterstrichen werden. Nach der Reduktion auf den Bewusstseinsstrom bringt Husserl „die radikalste Reduktion"50 auf die lebendige Gegenwart hervor: „[...] ich muss die phänomenologische Reduktion nicht abschliessen damit, dass ich die Welt einklammere [...], sondern auf mich als transzendentales Leben zurückge-

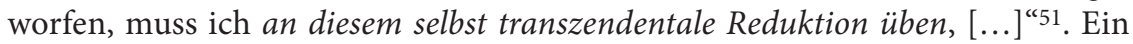
analoges zwiefaches Verfahren gilt auch für Heidegger. Ebenso wird „[d]ie phänomenologische Reduktion als die Rückfrage des Blickes vom Seiendem zu Sein“52, wie sie durch Heidegger im Jahr 1927 charakterisiert wird, durch einen Sprung vom Sein zum Ereignis nachgefolgt. Nach Heidegger vollzieht sich das Denken

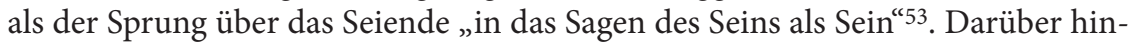

45 Es handelt sich um „[d]ie Er-eignung des Da-seins durch das Seyn und die Gründung der Wahrheit des Seins im Da-sein“ (Heidegger M., Beiträge zur Philosophie, op. cit., S. 262). „Dieser Gegenschwung des Brauchens und Zugehörens macht das Seyn als Ereignis aus, und die Schwingung dieses Gegenschwunges in der Einfachheit des Wissens zu heben und in seiner Wahrheit zu gründen ist das Erste, was uns denkerisch obliegt." (Ibid., S. 251).

Heidegger M., Unterwegs zur Sprache (1950-1959), hrsg. von Herrmann F.-W. von, Frankfurt am Main, Vittorio Klostermann, Gesamtausgabe 12, 1985, S. 247.

Ibid., S. 269. Vgl. Husserl E., Zur phänomenologischen Reduktion, op. cit., S. 183.

Husserl E., Zur phänomenologischen Reduktion, op. cit., S.187.

Ibid., S. 299 f.

52 Heidegger M., Die Grundprobleme der Phänomenologie, hrsg. von Herrmann F.-W. von, Frankfurt am Main, Vittorio Klostermann, Gesamtausgabe 24, 1975, S. 29.

53 Heidegger M., Der Satz vom Grund (1955-1956), hrsg. von Petra Jaeger, Frankfurt am Main, Vittorio Klostermann, Gesamtausgabe 10, 1997, S. 90. 
aus versucht er, „die Wahrheit des Seyns aus dessen eigenem Wesen zu fassen (Ereignis) “54. Dies führt zum weiteren Sprung, „der sich vom Sein als dem Grund des Seienden absetzt und so in den Abgrund springt " ${ }^{\text {55 }}$. Dieser Abgrund ist das Ereignis: „Sein als gründendes hat keinen Grund, spielt als der Ab-Grund jenes Spiel, das als Geschick uns Sein und Grund zuspielt" ${ }^{\star 56}$.

\section{Die Gegend und die Horizonthaftigkeit}

Nach der Darstellung vom Thema des Unscheinbaren in Husserl und Heidegger soll nun auf dem Begriff der Horizonthaftigkeit eingegangen werden. Wie Heidegger in seinem Aufatz „Mein Weg in die Phänomenologie“ sagt, betrachtet er die Phänomenologie nicht als eine Richtung, sondern als eine „bleibende Möglichkeit des Denkens, dem Anspruch des zu Denkenden zu entsprechen "57. Es handelt sich um eine Möglichkeit, die immer neu und ursprünglicher erfasst werden kann: „Wird die Phänomenologie so erfahren und behalten, dann kann sie als Titel verschwinden zugunsten der Sache des Denkens, deren Offenheit ein Geheimnis bleibt" 58 . Dementsprechend ist Heideggers Absicht hinsichtlich der Horizonthaftigkeit, wie er in einem der Feldweg-Gespräche sagt, „,nach dem Wesen des Horizontes und der möglichen Bestimmung dieses Wesens " ${ }^{\circ 9} \mathrm{zu}$ fragen. In dieser Hinsicht springen Unterschiede ins Auge, die sich mit der Unbestimmtheit der Horizonte, bzw. mit der Offenheit und dem Geheimnis verbinden.

Erstens geht es um die Situation und die Reichweite der Unbestimmtheit. Während die Welt als universaler Horizont und die einzelnen Horizonte sich für Husserl sozusagen auf demselben Niveau entfalten, hat Heidegger den verschiedenen Horizonten eine abhängige Stelle zugeschrieben. Jeder antizipierte Sinnesrahmen setzt ein ursprünglicheres Gebiet voraus. Nach Heidegger bietet das transzendentale Denken eine Aussicht, bei der die Gegenstände durch ein bestimmtes Aussehen ergriffen und festgehalten sind. Der Horizont sei ein Gesichtkreis, der einerseits über die einzelnen Gegenstände hinausgehe und andererseits die Aus-

\footnotetext{
Heidegger M., Beiträge zur Philosophie, op. cit., S. 250.

Heidegger M., Identität und Differenz (1955-1957), hrsg. von Friedrich-Wilhelm von Herrmann, Frankfurt am Main, Vittorio Klostermann, Gesamtausgabe 11, 2006, S. 48.

Heidegger M., Der Satz vom Grund, op. cit., S. 169.

Heidegger M., Zur Sache des Denkens, op. cit., S. 101.

Idem.

Heidegger M., Feldweg-Gespräche (1944-45), hrsg. von Schüßler I., Frankfurt am Main, Vittorio Klostermann, Gesamtausgabe 77, 1995, S. 105.
} 
sicht z. B. das Baumartige des Baumes, bei der er erscheine, umkreise. ${ }^{60}$ Der Horizont sei „ein Offenes“, weil er ein Rahmen für das Erscheinen von Gegenständen zeichne. Aber seine Offenheit sei „die uns zugekehrte Seite eines uns umgebenden Offenen, $[\ldots]^{\text {“61. }}$. Dieses umgebende Offene sei nicht anderes als „die Gegend aller Gegenden“, die sich als „das, was uns entgegenkommt" und „das Öffnende des Offenen"62 zeige. M.a.W.: der Horizont sei aus einer ursprünglichsten Gegend als ein Gesichtkreis für unseres Vorstellen zu verstehen. Jenseits der Darstellung der Gegend auf Grund von ihrem Verhältnis zu uns mittels des Horizontes müsse das, „was das uns umgebende Offene in sich ist" ${ }^{\text {63 }}$ gesucht werden.

Heidegger identifiziert den Horizont mit einer Struktur der Begrenzung. In der Vorlesung des Sommersemesters 1927 bemerkte er schon: „Aber Horizont, von ó $\rho \zeta \varepsilon ı v,[\ldots]$ besagt einfach an sich das Eingrenzende, das Umschließende,

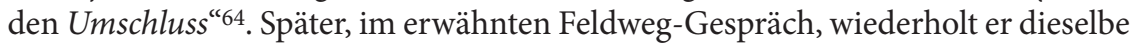
Betrachtung und setzt jenseits des Horizontes die Gegend, aus der die Offenheit hervorkommt. Der Horizont könnte nicht als „das Umgrenzende“ gesehen werden, wenn er grenzenloser sei, weil er wie „eine ungerade Gerade“, d.h. „eine bare Unmöglichkeit" 65 sein würde. Deshalb sei eine unendliche Folge von ineinander implizierten Horizonten unbegreiflich.

Grundsätzlich anders steht es bei Husserl. Wenn die Horizonte gemä $\beta$ ihrer offenen Unbestimmtheiten, die sich über alle Bestimmtheit und jenseits ihrer ausbreitet, betrachtet werden, verschwindet der Kontrast zwischen zwei Arten von Offenheit. Ein Horizont der Unbestimmtheit als das Andere der Vorbekanntheit wird nicht nur innerhalb der Struktur der Bestimmtheit des Horizontes, sondern auch jenseits jeder Antizipation eingeschlossen. Husserl sagt, dass der Strom des Lebens „seine lebendige, aber strömend sich ändernde Reichweite hat - ohne eigentlich Grenze und doch begrenzt und veränderlich begrenzt "66. Die Begrenzung stimmt mit einer Unbegrenztheit des Lebens, d.h. seiner Horizonte überein. Für Husserl gibt es kein „Andere[s] des transzendental Horizontalen “67, weil beide Arten von

„Diese Aussicht umgibt als Gesichtskreis das wahrnehmende Vorstellen von einzelnen Gegenständen. Sie ist der Horizont dieser Gegenstände und des auf sie gerichteten Wahrnehmens [...].“ (Ibid., S. 98).

Ibid., S. 112.

Ibid., S. 113, 117.

Ibid., S. 113. Vgl. Figal G., op. cit., S. 258-268.

64 Heidegger M., Metaphysische Anfangsgründe der Logik im Ausgang von Leibniz, hrsg. von Held K., Frankfurt am Main, Vittorio Klostermann, Gesamtausgabe 26, 1978, S. 269.

Heidegger M., Feldweg-Gespräche, op. cit., S. 94.

66 Husserl E., Erste Philosophie. Zweiter Band, op. cit., S. 467.

Heidegger M., Feldweg-Gespräche, op. cit., S. 102. 
Offenheit - Offenheit in Bezug auf die besonderen Horizonte und Offenheit in Bezug auf den universalen Horizont - eine transzendentale Bedeutung haben.

Ein zweiter Unterschied liegt in Heideggers Kennzeichnung der Offenheit. Während Husserl die Horizonthaftigkeit gemä $\beta$ Gegensätzen wie Fülle/Leere und Bestimmtheit/Unbestimmtheit analysiert, zeigt die Gegend Kontraste, die durch ihre Fülle und Bestimmtheit charakterisiert sind. Dazu bemerkt Heidegger: „Überall wo ein Anwesendes anderem Anwesenden entgegen kommt oder auch nur entgegen verweilt, [...] da waltet schon Offenheit, ist freie Gegend im Spiel“"68. Diese Überlegung zeigt, dass die Offenheit aus einer wechselseitigen Beziehung zwischen den Dingen innerhalb eines ursprünglichen Zusammenhanges besteht. Das Terminus „Gegend“ ist mit den Worten „entgegenkommen“, „begegnen“ und "gegenüber“ verwandt. Die Dinge kommen entgegen und diese Entgegnung ist der Grund dafür, dass die Offenheit der Gegend sich mit keiner unbestimmten Leere, sondern mit einem Gefüge von Bezügen verbindet. Deshalb ist die Gegend, wenn man es so ausdrücken will, wie die Masse einer geschlossenen Kugel. Heidegger weist auf eine „Einfachheit, die nicht Leere, sondern Grund der Fülle ist, die aus der Ent-gegnung als Streit entspringt ${ }^{\text {" } 69}$ hin. Das Gegenteil des bestimmten Gefüges ist kein offenes und unbestimmtes Netz von Verweisungen, sondern ein Riss innerhalb einer Versammlung. Es geht nicht um eine Kluft, weil der Riss nicht

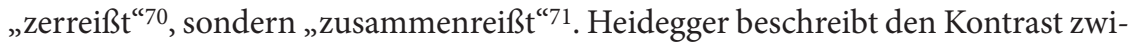
schen der mit der Unbestimmtheit verbundenen Leere und der mit dem Inhalt eines Gefüges erfüllten Leere auf diese Weise: „Die Offenheit des Lichtens der Verbergung ist daher ursprünglich keine bloße Leere des Unbesetztseins, sondern die gestimmt stimmende Leere des Ab-grundes, der [...] ein gestimmter und d.h. hier gefügter ist“72.

Der dritte wesentliche Unterschied liegt darin, dass die Unbestimmtheit bei Husserl das Erfahrene umfasst und durchdringt. Im Gegensatz dazu behandelt Heidegger das Problem in der Sphäre der Sprache. Weil der Horizont ein Umschluss ist und die Gegend aller Gegenden keine unbestimmte Leere ist, wendet er sich der Sage zu, die nicht das Denken im Sinne des begrifflichen Vorstellens ausdrückt, sondern als ein „Zuwinken“ und „Abwinken“ angesehen wird. ${ }^{73}$ Heidegger

\footnotetext{
Heidegger M., Zur Sache des Denkens, op. cit., S. 80.

Heidegger M., Beiträge zur Philosophie, op. cit., S. 471.

Heidegger M., Unterwegs zur Sprache, op. cit., S. 24.

Heidegger M., Holzwege, op. cit., S. 51.

Heidegger M., Beiträge zur Philosophie, op. cit., S. 381.

„Im Begreifen liegt nämlich die Verhaltensweise eines Inbesitznehmens. Der griechische ó dagegen umgibt stark und zart das, was das Sehen in den Blick nimmt; er begreift nicht." (Heidegger M., Seminare, op. cit., S. 399).
} 
findet keine Komponente der Erfahrung, die Rechenschaft über einen Entzug oder seine Verborgenheit ablegt. Aus diesem Grund schildert er die Sage als ein Zeigen,

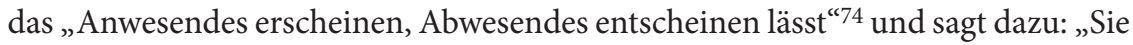
befreit Anwesendes in sein jeweiliges Anwesen, entfreit Abwesendes in seinem jeweiligen Abwesen "75. Heidegger weist dem Winken und den Tautologien die Funktion zu, die für Husserl der Horizont der Unbestimmtheit dank seiner Unbegrenzheit erfüllt, d.h., die Funktion, uns zu den Dingen mit einer Verwahrung der Tiefe seiner Verbergung zu verhalten, so dass wir kein Besitz von ihnen ergreifen können. Über die tautologischen Sätze wie „Die Welt weltet“ oder „Die Sprache spricht“ äussert sich Heidegger folgendermaßen: „Das Eigentümliche der Sätze dieser Art liegt darin, dass sie nichts sagen und zugleich das Denken aus der höchsten Entschiedenheit an seine Sache binden "76. Angesichts dieser Sätze spricht er von „dem Grenzenlosen, in das sie den Auftrag des Denkens verweisen"77. Husserl bewahrt diese Grenzenlosigkeit mit der Unbestimmtheit der Horizonthaftigkeit, weil die Latenz durch keine Vervollkommnung der Vergegenständlichung beiseitegelassen werden kann und immer als das Unerfahrene die erfahrene Gegenständlichkeit begleitet. Die von Heidegger hervorgehobene „Verwahrungslosigkeit“ lässt sich vermeiden, wenn die Unmöglichkeit einer Gesamterfahrung unterstrichen wird. Dies bedeutet, dass es möglich ist, eine vergegenständlichte Sprache zu benutzen, ohne in eine Machenschaft zu verfallen. Infolge der Unbestimmtheit der Horizonthaftigkeit bringt Husserl eindringlich und deutlich die Möglichkeit der Verwahrung der Dinge ans Licht. Gegen Heideggers Meinung über das Geheimnis sagt er: „Die Dinge ihrem Sein und Wesen nach haben kein Geheimnis, oder besser keine Mysterien; sie sind nur nicht völlig bekannt" ${ }^{\text {"78. }}$.

Roberto Walton ist Professor an der Universität Buenos Aires. Veröffentlichungen: Mundo, conciencia y temporalidad (Buenos Aires, 1993); El fenómeno y sus configuraciones (Buenos Aires, 1993); Introducción al pensar fenomenológico (Buenos Aires, 2013); Intencionalidad y horizonticidad (Bogotá, 2015); Horizonticidad e historicidad (Bogotá, im Druck).

\footnotetext{
Heidegger M., Unterwegs zur Sprache, op. cit., S. 246.

Idem.

Heidegger M., Was heisst Denken?, op. cit., S. 157.

Idem.

Husserl E., Zur Phänomenologie der Intersubjektivität. Texte aus dem Nachlass. Erster Teil: 19051920, hrsg. von Kern I, Den Haag, Martinus Nijhoff, Husserliana XIII, 1973, S. 10.
} 